\title{
ESTIMATIVAS DE CORRELAÇÃO E REPETIBILIDADE PARA CARACTERES DO FRUTO DE BACURIZEIRO
}

\author{
Estimates correlation and repeatability for characters of the bacurizeiro fruit \\ João Tomé de Farias Neto ${ }^{1}$, José Urano de Carvalho ${ }^{1}$, Carlos Hans Muller ${ }^{1}$
}

\begin{abstract}
RESUMO
Conduziu-se este trabalho com o objetivo de estimar os coeficientes de correlação e de repetibilidade envolvendo doze caracteres do fruto de bacurizeiro: comprimento do fruto (CF), diâmetro do fruto (DF), espessura da casca (EC), peso da casca (PC), peso das sementes (PS), número de sementes (NS), volume da cavidade interna (VCI), peso do fruto (PF), peso total de polpa (PTP), porcentagens de casca $(\% \mathrm{C})$, sementes $(\% \mathrm{~S})$ e polpa total $(\% \mathrm{PTP})$. As estimativas dos coeficientes de repetibilidade das características CF, DF, EC, PC, PS, VCI, PTP, \%C e \%PTP demonstraram alta regularidade nos indivíduos, e quatro a doze medições são necessárias para obter predições com confiabilidade em torno de $95 \%$. Com exceção do caráter EC, não foram observadas grandes diferenças entre as estimativas dos coeficientes de repetibilidade obtidos pelo método da ANOVA e pelos métodos de análise multivariada. O aumento na precisão em termos de predição do valor real dos indivíduos além de 95\% implicará aumentar, sobremaneira, o número de medições, que, por sua vez, pouco acrescentará em termos de precisão, sendo, então, injustificado seu uso. PTP apresentou associações positivas com o DF, VCI e PF.
\end{abstract}

Termos para indexação: Platonia insignis, herdabilidade, fruticultura, caracterização, acesso.

\section{ABTRACT}

This work had as aim to estimate the repeatability and correlation coefficients involving twelve characters of the bacurizeiro fruit: length of the fruit (CF), diameter of the fruit (DF), thickness of the rind (EC), weight of the rind (PC), weight of the seeds (PS), number of seeds (NS), volume of internal cavity (VCI), weight of the fruit (PF), total weight of pulp (PTP), percentages of rind $(\% \mathrm{C})$, seeds $(\% \mathrm{~S})$ and total pulp $(\% \mathrm{PTP})$. The estimates of the coefficients of repeatability of characteristics CF, DF, EC, PC, PS, VCI, PTP, \%C and \%PTP demonstrated high regularity in the individuals and four to twelve measurements are necessary to get predictions with around $95 \%$ of reliability. With exception of character EC, had not been observed great differences between the estimates of the coefficients of repeatability obtained by the ANOVA method and the methods of multivaried analysis. The increase in the precision in terms of prediction of the real value of the individuals beyond $95 \%$, will imply to excessive increase, in the number of measurements adding little in precision terms, being then unjustifiable its use. Total weight of pulp presented positive associations with the diameter of fruit, volume of the internal cavity and weight of the fruit.

Index terms: Platonia insignis, heritability, fruit crop, characterization, access.

(Recebido para publicação em 17 de junho de 2003 e aprovado em 27 de outubro de 2003)

\section{INTRODUÇÃO}

O bacurizeiro (Platonia insignis Mart.) é uma espécie frutífera e madeireira nativa da Amazônia e com centro de origem no Estado do Pará, onde estão estabelecidas densas e diversificadas populações naturais. É também encontrado, espontaneamente, nos outros Estados da Amazônia brasileira, no Piauí e Maranhão (LOUREIRO et al., 1979; CAVALCANTE, 1996). Os frutos dessa Clusiaceae têm grande aceitação nas áreas de ocorrência natural da espécie, principalmente no Estado do Pará, que se constitui no maior produtor e principal consumidor. Estimativas indicam que somente na cidade de Belém, PA são comercializados, anualmente, sete milhões de frutos, com valor total de U\$ 1,61 milhão (SHANLEY, 2000).

$\mathrm{Na}$ experimentação com espécies perenes, temse realizado, na seleção da espécie ou do genótipo superior, avaliações periódicas. Essas avaliações com freqüência envolvem grande número de experimentos, várias etapas e diferentes características, significando o emprego de considerável mão-de-obra e tempo.

Como alternativa para sobrepujar tais limitações, o conhecimento do coeficiente de repetibilidade (r) torna-se necessário. Por meio da estimativa desse coeficiente, é possível determinar quantas medições deverão ser realizadas em cada indivíduo para que a avaliação e/ou caracterização fenotípica seja feita com a 
precisão desejada pelo pesquisador. Valores altos da estimativa da repetibilidade indicam que é possível predizer o valor real dos indivíduos com número relativamente pequeno de avaliações (CRUZ e REGAZI, 1994). Adicionalmente, a repetibilidade expressa o valor máximo que a herdabilidade pode atingir, pois expressa a proporção da variância fenotípica que é atribuída às diferenças genéticas confundidas com os efeitos permanentes que atuam na cultivar ou progênie. Assim, a utilização do coeficiente de repetibilidade associado à propagação vegetativa consiste em uma estratégia de melhoramento para o bacurizeiro, a qual, quando aplicada ao germoplasma atualmente disponível, permitirá ganhos genéticos rápidos, comparados aos obtidos por estratégias baseadas em teste de progênies.

Outro parâmetro importante no melhoramento de plantas visando ao aumento da eficiência da seleção é o conhecimento das correlações entre os caracteres de interesse agronômico, porquanto permite direcionar as estratégias de melhoramento a serem adotadas, maximizando os ganhos genéticos por meio dos ciclos de seleção. Cruz e Regazi (1994) ressaltaram a importância das correlações, afirmando que elas quantificam a possibilidade de ganhos via seleção indireta por seleção em caracteres correlacionados e que caracteres correlacionados de baixa herdabilidade têm a seleção mais eficiente quando realizada sobre caracteres que lhe são correlacionados. Objetivou-se com este trabalho obter estimativas para os coeficientes de correlação e de repetibilidade de doze caracteres dos frutos de bacurizeiro.

\section{MATERIAL E MÉTODOS}

Foram utilizados dados de caracterização de frutos de 13 matrizes de bacurizeiro estabelecidas em uma população natural, no município de Carutapera, MA. Em cada indivíduo foram amostrados dez frutos e avaliaram-se as seguintes características: comprimento do fruto (CF), diâmetro do fruto (DF), espessura da casca (EC), peso da casca (PC), peso das sementes (PS), número de sementes (NS), volume da cavidade interna (VCI), peso do fruto (PF), peso total de polpa (PTP), porcentagens de casca $(\% \mathrm{C})$, sementes $(\% \mathrm{~S})$ e polpa total (\% PT).

Os caracteres CF, DF e a EC foram determinados com paquímetro digital, sendo o CF medido na porção mais larga do fruto; DF compreendeu a distância entre as cicatrizes do pedúnculo e do estigma e EC foi medida após a abertura transversal dos frutos no ponto médio da distância entre as referidas cicatrizes.
O VCI foi estimado medindo-se o volume de água comportado na parte interna da casca, após a abertura transversal dos frutos e remoção das sementes, polpa e do conjunto representado pela coluna placentária e óvulos abortados. O valor do caráter NS foi transformado para $\sqrt{x}$ e as características $\% \mathrm{C}, \% \mathrm{~S}$ e $\% \mathrm{PT}$ foram transformadas para arcosin da raiz quadrada da proporção $\left(\arcsin (\mathrm{x} / 100)^{1 / 2}\right)$, conforme Steel e Torrie (1980).

A estimativa dos coeficientes de repetibilidade foi obtida pelos métodos: análise de variância (ANOVA), componentes principais e análise estrutural, com base na matriz de correlação e de covariância. Esses métodos encontram-se descritos em Abeywardena (1972), Rutledge (1974), Mansour et al. (1974) e Cruz e Regazi (1994). Todas as análises estatísticas foram realizadas utilizando o programa computacional GENES (CRUZ, 2001).

Estimativas de correlação fenotípica entre todos os caracteres foram realizadas usando-se as fórmulas apresentadas por Falconer (1987). Para testar se os valores das correlações não diferiram de zero, utilizou-se o teste $\mathrm{t}$, com n-2 número de graus de liberdade, sendo n o número de genótipos.

\section{RESULTADOS E DISCUSSÃO}

Os resultados da análise de variância, utilizando-se o modelo com um fator de variação, são apresentados na Tabela 1. Com exceção do caráter espessura da casca (EC), cujo valor de F não foi significativo, foram detectadas diferenças significativas, a $1 \%$ de probabilidade, para todas as outras, evidenciando a presença de variabilidade das características entre os indivíduos. Os valores médios estimados para a maioria dos caracteres estudados foram semelhantes aos encontrados por Guimarães et al. (1992). Carvalho et al. (2003) também obtiveram valores semelhantes, com exceção dos caracteres NS $(2,3 \pm 0,7), \% \mathrm{~S}(18,7 \pm 3,3)$ e $\% \mathrm{PTP}(27,7 \pm 2,4)$. Os maiores coeficientes de variação foram estimados para PF $(37,6 \%)$, PS $(36,5 \%)$, NS $(33,5 \%)$, PTP $(31,4 \%)$ e VCI $(21,5 \%)$.

$\mathrm{Na}$ Tabela 2, apresentam-se as estimativas do coeficiente de repetibilidade obtidas pelos diferentes procedimentos estatísticos. Com exceção do caráter EC, houve concordância muito grande nas magnitudes dos coeficientes de repetibilidade obtidas pelos diferentes métodos, conferindo-lhes maior confiabilidade. 
TABELA 1 - Análise de variância para comprimento do fruto (CF), diâmetro do fruto (DF), espessura da casca (EC), peso da casca (PC), peso da semente (PS), número de sementes (NS), volume da cavidade interna (VCI), peso do fruto $(\mathrm{PF})$, peso total de polpa $(\mathrm{PTP})$, porcentagem de casca $(\% \mathrm{C})$, semente $(\% \mathrm{~S})$ e peso total de polpa (\%PTP). Belém, PA. 2002.

\begin{tabular}{ccccccccccccc}
\hline FV & CF & DF & EC & PC & PS & NS & VCI & PF & PTP & \% C & \%S & \%PTP \\
\hline Indiv. & $16,1^{* *}$ & $8,15^{* *}$ & 0,32 & $75235^{* *}$ & $8065^{* *}$ & $9,2 * *$ & $19111^{* *}$ & $381,2^{* *}$ & $4174 * *$ & $54,4^{* *}$ & $33,5^{* *}$ & $98,2^{* *}$ \\
Resíduo & 0,398 & 0,391 & 0,80 & 1409,1 & 554,79 & 1,3 & 711,51 & 44,52 & 332,04 & 4,19 & 6,60 & 8,64 \\
\hline Média & 9,67 & 8,31 & 1,12 & 226 & 64,6 & 3,4 & 123,8 & 348,6 & 58,0 & 53,50 & 24,86 & 24,3 \\
CV $(\%)$ & 6,5 & 6,9 & 25,7 & 16,5 & 36,5 & 33,5 & 21,5 & 37,6 & 31,4 & 3,83 & 10,33 & 12,11 \\
\hline
\end{tabular}

**Significativo a $1 \%$ de probabilidade pelo teste $\mathbf{F}$.

Percebe-se que, para os caracteres CF, DF, PC e VCI, a repetibilidade foi relativamente alta (acima de $0,70)$ em todos os métodos de estimação, com o valor real em torno de $97,4 \%$, o que pode ser considerado um ótimo valor. Para os caracteres PS, PTP, \%C e \%PTP, a repetibilidade foi pouco menor, porém variando de 0,510 a 0,700 , com predição do valor médio real em torno de $93,6 \%$, o que pode ser considerado bastante satisfatório. As menores estimativas foram obtidas para os caracteres NS, PF e \%S, que variaram entre 0,289 a 0,479 , com a predição do valor real médio de $87,7 \%$, sugerindo a necessidade de aumento de medições para o aumento da acurácia, que certamente implicará maiores custos e emprego e mão-de-obra. Esse fato também demonstra que essas características são mais influenciadas pelo ambiente, e que a seleção indireta via características de melhor controle genético seria uma estratégia interessante a ser aplicada.

A característica EC apresentou discrepâncias nas estimativas do coeficiente de repetibilidade obtidas pelas metodologias da ANOVA e análise estrutural, 0,288 e 0,224 , respectivamente, e demais metodologias, cujas estimativas foram substancialmente mais altas. Portanto, ambos os métodos foram ineficientes para eliminar o efeito de variação da $\mathrm{EC}$, que ocorreu entre os indivíduos e se tornou um componente adicional do erro experimental.

$\mathrm{Na}$ Tabela 3, encontram-se as estimativas do número de medições necessárias para se ter diferentes valores de predição do valor real, obtidos a partir dos coeficientes de repetibilidade estimados pelo método dos componentes principais, baseado na matriz de correlação. Percebe-se que, para os caracteres CF, DF, EC, PC, PS, VCI, PTP, \%C e \%PTP, são necessárias de quatro a 12 medições, para obter predições com confiabilidade em torno de $95 \%$. Para o mesmo nível de confia- bilidade, são necessárias 25, 29 e 33 medições para PF, $\mathrm{NS}$ e $\% \mathrm{~S}$, respectivamente, que são as características que apresentaram menores coeficientes de repetibilidade (Tabela 2). Nota-se que é possível obter maiores níveis de precisão para essas características mediante maior número de medições, nas quais se espera alcançar $99 \%$ de determinação do valor real dos indivíduos. Entretanto, o processo seletivo seria impraticável para a avaliação de alguns caracteres, pois seria necessário realizar 126, 149 e 169 medições para PF, NS e \%S, respectivamente.

As estimativas de correlação podem ser úteis quando determinado caráter de interesse é de difícil avaliação. Nessa situação, o processo seletivo pode se tornar mais simples se esse caráter apresentar alta correlação positiva com outro de fácil avaliação, haja vista que, nesse caso, aumentos em um caráter tendem a ser acompanhados de aumentos no outro e vice-versa, não necessitando de adoções de restrições na seleção para obtenção de ganhos no sentido desejado. As estimativas de correlações fenotípicas entre 12 caracteres são apresentadas na Tabela 4. A estimativa positiva e elevada de correlação entre peso do fruto e peso de semente $(0,889)$ indica que frutos de maior peso representam sementes maiores. Portanto, a seleção de plantas com fruto grande e semente pequena é bastante dificultada. Os caracteres referentes ao tamanho do fruto (comprimento e diâmetro) apresentaram estimativas positivas com o peso total da polpa, sugerindo que frutos maiores estão associados com maior quantidade de polpa, o que já era esperado e desejável. A correlação entre peso total de polpa e peso do fruto apresenta valor positivo $(0,643)$, fato que assegura que a seleção de frutos grandes, principalmente de maior diâmetro, resulte em maior quantidade de polpa. 
TABELA 2 - Estimativas da repetibilidade para comprimento do fruto (CF), diâmetro do fruto (DF), espessura da casca (EC), peso da casca (PC), peso da semente (PS), número de sementes (NS), volume da cavidade interna (VCI), peso do fruto (PF), peso total de polpa (PTP), porcentagens de casca $(\% \mathrm{C})$, sementes $(\% \mathrm{~S})$ e peso total de polpa (\%PTP). Belém, PA.2002.

\begin{tabular}{cccccc}
\hline \multirow{2}{*}{ Caráter } & \multirow{2}{*}{ Anova } & \multicolumn{2}{c}{ Componentes principais } & \multicolumn{2}{c}{ Análise estrutural } \\
\cline { 3 - 6 } & & Covariância & Correlação & Covariância & Correlação \\
\hline CF & $0,798(97,4)^{1}$ & $0,813(97,5)$ & $0,804(97,6)$ & $0,802(97,6)$ & $0,790(97,4)$ \\
DF & $0,698(95,8)$ & $0,714(96,1)$ & $0,715(96,2)$ & $0,711(96,1)$ & $0,698(95,8)$ \\
EC & $0,228(74,6)$ & $0,625(94,3)$ & $0,754(96,8)$ & $0,656(95,0)$ & $0,224(74,3)$ \\
PC & $0,839(98,1)$ & $0,858(96,3)$ & $0,857(98,4)$ & $0,856(98,3)$ & $0,838(98,1)$ \\
PS & $0,574(93,1)$ & $0,674(95,3)$ & $0,639(94,5)$ & $0,624(94,3)$ & $0,569(92,9)$ \\
NS & $0,377(85,8)$ & $0,431(89,1)$ & $0,400(86,9)$ & $0,377(85,8)$ & $0,375(85,7)$ \\
VCI & $0,721(96,2)$ & $0,748(96,7)$ & $0,734(96,5)$ & $0,731(96,4)$ & $0,712(96,1)$ \\
PF & $0,431(88,3)$ & $0,479(90,1)$ & $0,441(88,7)$ & $0,433(88,4)$ & $0,433(88,4)$ \\
PTP & $0,536(92,0)$ & $0,652(94,9)$ & $0,701(96,1)$ & $0,603(93,8)$ & $0,547(92,3)$ \\
\% C & $0,544(92,3)$ & $0,622(94,3)$ & $0,700(96,0)$ & $0,695(95,8)$ & $0,554(92,5)$ \\
\%S & $0,289(80,3)$ & $0,395(86,7)$ & $0,369(85,4)$ & $0,294(80,7)$ & $0,296(80,8)$ \\
\%PTP & $0,510(91,2)$ & $0,594(93,6)$ & $0,659(95,1)$ & $0,619(94,2)$ & $0,527(91,8)$ \\
\hline
\end{tabular}

${ }^{1}$ Valores referentes ao coeficiente de determinação associado ao coeficiente de repetibilidade.

TABELA 3 - Estimativas do coeficiente de repetibilidade pelo método de componentes principais (correlação) e número de medições, associado a vários coeficientes de determinação $\left(\mathrm{R}^{2}\right)$, para comprimento do fruto $(\mathrm{CF})$, diâmetro do fruto (DF), espessura da casca (EC), peso da casca (PC), peso da semente (PS), volume da cavidade interna (VCI), número de sementes (NS), peso da placenta (PP), peso da polpa aderida (PPA), peso do fruto (PF), número segmentos partenocárpicos (NSP), peso dos segmentos partenocárpicos (PSP), peso total de polpa (PTP), rendimento de PTP. Belém, PA. 2002.

\begin{tabular}{ccccc}
\hline Caracteres & Repetibilidade & $\mathbf{R}^{\mathbf{2}}=\mathbf{0 , 9 0}$ & $\mathbf{R}^{\mathbf{2}} \mathbf{= 0 , 9 5}$ & $\mathbf{R}^{\mathbf{2}}=\mathbf{0 , 9 9}$ \\
\hline CF & 0,8047 & 3 & 5 & 25 \\
DF & 0,7153 & 4 & 8 & 40 \\
EC & 0,7545 & 3 & 7 & 33 \\
PC & 0,8571 & 2 & 4 & 17 \\
PS & 0,6329 & 6 & 12 & 58 \\
NS & 0,4008 & 14 & 29 & 149 \\
VCI & 0,7337 & 4 & 7 & 36 \\
PF & 0,4409 & 12 & 25 & 126 \\
PTP & 0,7143 & 4 & 8 & 40 \\
$\%$ C & 0,7070 & 4 & 8 & 41 \\
$\%$ \% & 0,3696 & 16 & 33 & 169 \\
$\%$ PTP & 0,6592 & 5 & 10 & 52 \\
\hline
\end{tabular}


TABELA 4 - Estimativas dos coeficientes de correlação fenotípica entre os caracteres comprimento do fruto (CF), diâmetro do fruto (DF), espessura da casca (EC), pesos da casca (PC), semente (PS), número de sementes/fruto (NS), volume cavidade interna (VCI), peso fruto (PF), peso total de polpa (PTP), porcentagens de casca (\%C), semente $(\%$ S) e peso total de polpa (\%PTP). Belém, PA. 2002.

\begin{tabular}{cccccccccccc}
\hline Caract & DF & EC & PC & PS & NS & VCI & PF & PTP & \% C & \% S & \%PTP \\
\hline CF & 0,559 & 0,268 & 0,628 & 0,619 & 0,605 & 0,702 & 0,635 & 0,389 & $-0,018$ & 0,404 & $-0,293$ \\
& & 0,359 & 0,863 & 0,807 & 0,662 & 0,863 & 0,870 & 0,609 & $-0,084$ & 0,415 & $-0,266$ \\
EC & & & 0,456 & 0,342 & 0,286 & 0,327 & 0,418 & 0,216 & 0,272 & 0,045 & $-0,251$ \\
PC & & & & 0,864 & 0,707 &, 894 & 0,970 & 0,545 & 0,212 & 0,359 & $-0,450$ \\
PS & & & & 0,848 & 0,90 & 0,889 & 0,397 & $-0,011$ & 0,676 & $-0,534$ \\
NS & & & & & 0,771 & 0,747 & 0,348 & $-0,063$ & 0,710 & $-0,454$ \\
VCI & & & & & & 0,933 & 0,656 & $-0,123$ & 0,515 & $-0,271$ \\
PF & & & & & & & & 0,053 & 0,403 & $-0,345$ \\
PTP & & & & & & & & $-0,454$ & $-0,011$ & 0,450 \\
\% C & & & & & & & & & & $-0,171$ & $-0,641$ \\
\% S & & & & & & & & & & & $-0,542$ \\
\hline
\end{tabular}

Coeficientes acima de 0,251 são significativos a $1 \%$ de probabilidade pelo teste $t$.

A correlação significativa e negativa entre \% PTP e \%C (-0,641) e \%PTP com \%S (-0,542), aliada ao bom índice de repetibilidade apresentado pelo primeiro $(0,659)$, indica que a seleção para o primeiro resultará na obtenção de progênies, as quais tenderiam a ter menor $\% \mathrm{C}$ e $\% \mathrm{~S}$. Resultados semelhantes foram obtidos por Guimarães et al. (1992), que observaram que o rendimento de polpa está associado com o menor peso de sementes e casca do que com o tamanho do fruto. De fato, Carvalho et al. (2003), estudando um tipo de bacuri com elevado rendimento porcentual de polpa, verificaram que as principais características diferencias relativamente a outros tipos encontrados em populações naturais e em áreas de cultivo são: casca fina, elevado número de segmentos partenocárpicos e grande freqüência de frutos com, no máximo, duas sementes. Ainda segundo esses autores, o tamanho, peso e volume da cavidade interna dos frutos são semelhantes ao da maioria dos tipos ocorrentes em área de dispersão e em áreas de cultivo.

\section{CONCLUSÕES}

Os coeficientes de repetibilidade de $\mathrm{CF}, \mathrm{DF}$, EC, PC, PS, VCI, PTP, \%C e \%PTP demonstram alta regularidade nos indivíduos, e 4 a 12 medições são necessárias para obter predições com confiabilidade em torno de $95 \%$. Considerando o mesmo nível de precisão, são necessárias 25, 29 e 33 para PF, NS e \%C, respectivamente.

Não foram observadas grandes diferenças entre os coeficientes de repetibilidade obtidos pelo método da ANOVA e pelos métodos de análise multivariada, exceto para EC.

$\mathrm{O}$ aumento na precisão em termos de predição do valor real dos indivíduos além de 95\% implicará aumentar, sobremaneira, o número de medições que, por sua vez, pouco acrescentará em termos de precisão, sendo então injustificado seu uso.

Peso total de polpa apresentou associações positivas com o diâmetro de fruto, volume da cavidade interna e peso do fruto.

\section{REFERÊNCIAS BIBLIOGRÁFICAS}

ABEYWARDENA, V. An application of principal components analysis in genetics. Journal of Genetic, New York, v. 16, n. 9, p. 27-51, 1972.

Ciênc. agrotec., Lavras, v. 28, n. 2, p. 300-305, mar./abr., 2004 
CARVALHO, J. E. U.; NAZARÉ, R. F. R.; NASCIMENTO, W. M. O. Características físicas e fisícas-químicas de um tipo de bacuri (Platonia insignis Mart.) com rendimento industrial superior. Revista Brasileira de Fruticultura, Cruz das Almas, v. 25, n. 2, p. 326-328, 2003.

CAVAlCANTE, P. B. Frutas comestíveis da Amazônia. 6. ed. Belém: CNPq/Museu Paraense Emílio Goeldi, 1996. 279 p. (Coleção Adolpho Ducke).

CRUZ, C. D. Programa genes: aplicativo computacional em genética e estatística. Viçosa: UFV, 2001. 648 p.

CRUZ, C. D.; REGAZI, A. J. Modelos biométricos aplicados ao melhoramento genético. Viçosa: UFV, 1994. $390 \mathrm{p}$.

FALCONER, D. D. Introdução a genética quantitativa. Viçosa: UFV, 1987. 279 p.

GUIMARÃES, A. D. G.; MOTA, M. G. C.; NAZARÉ, R. F. R. Coleta de germoplasma de bacuri (Platonia insignis Mart.) na Amazônia. I: microrregião Campos do Marajó (Soure/Salvaterra). Belém: EMBRAPACPATU, 1992. 23 p. (Embrapa-CPATU. Boletim de Pesquisa, 132).

LOUREIRO, A. A.; SILVA, M. F. da; ALENCAR, J. da C. Essências madeireiras da Amazônia. Manaus: CNPq/INPA, 1979. v. 1, 245 p.

MANSOUR, H.; NORDHEIM, E. V.; RUTLEDGE, J. J. Estimation of repeatability. Theoretical and Applied. Genetics, Madison, v. 61, p. 247-250, 1974.

RUTLEDGE, J. J. A scaling which remove bias of Abeywardena's estimatior of repeatibility. Journal of Genetic, New York, v. 16, p. 247-254, 1974.

SHANLEY, P. As the forest falls: the changing use, ecology and value of non-timber forest resources for caboclo communities in eastern Amazonia. 2000. $214 \mathrm{f}$. Thesis (Doctor) - The Durrel Institute of Conservation and Ecology, The University of Kent, Cantebury, 2000.

STEEL, R. G.; TORRIE, J. H. Principles and procedures of statistic. 2. ed. New York: McGrawHill, 1980. 633 p. 